\title{
Universitarios y Confinamiento. Factores Socio- personales que Influyen en sus Niveles de Ansiedad y Empatía
}

\author{
University Students and Home Lockdown. Socio- \\ personal Factors that Influence their Levels of Anxiety \\ and Empathy
}

\author{
Tatiana Íñiguez-Berrozpe \\ Raquel Lozano-Blasco* \\ Alberto Quílez-Robres \\ Alejandra Cortés Pascual \\ Universidad de Zaragoza, España
}

\begin{abstract}
El cierre de los centros educativos, incluidas las universidades, y la orden de estado de alarma, con el consiguiente confinamiento de la población, ha afectado a todos los niños, adolescentes y jóvenes del territorio español. No obstante, mientras la comprensible preocupación por el estado emocional de los niños ha sido evidente por parte de las instituciones y la sociedad en general, menos atención ha recibido la salud mental de los jóvenes universitarios confinados. En el presente artículo exploramos los niveles de ansiedad y empatía de una muestra de $\mathrm{n}=124$ universitarios, residentes en Aragón, a partir de las escalas Beck-II Depression Inventory (BDI-II) y Baron-Cohen y Wheelwright's Empathy Quotient (EQ), y de los factores sociopersonales que les están influyendo durante el estado de alarma. Para ello, tras un análisis preliminar descriptivo y mediante correlaciones, aplicamos un modelo de ecuaciones estructurales (SEM). Los resultados muestran, por un lado, que el sexo del entrevistado, el tipo de hogar (personas con las que convive) y el aumento en el consumo de TV influyen en los niveles de ansiedad. Por otra parte, los niveles de empatía vienen condicionados por la situación laboral de la madre durante el confinamiento y el tener que ayudar a otras personas fuera del hogar.
\end{abstract}

Descriptores: Universitarios; COVID-19; Confinamiento; Ansiedad; Empatía.

The closure of educational centers, including universities, and the order of a state of alarm, with the consequent lockdown of the population, has affected all children, adolescents and young people in Spanish territory. However, while the understandable concern for the emotional state of children has been evident by institutions and society in general, less attention has received the mental health of confined university students. In this article we explore the levels of anxiety and empathy in a sample of $n=124$ university students, residents of Aragon, using the Beck-II Depression Inventory (BDI-II) and Baron-Cohen y Wheelwright's Empathy Quotient (EQ) scales), and the socio-personal factors that are influencing them during the state of alarm. To do this, after a preliminary descriptive analysis and through correlations, we apply a structural equation model (SEM). The results show, on the one hand, that the sex of the interviewee, the type of household (people with whom they live) and the increase in TV consumption influence anxiety levels. On the other hand, empathy levels are conditioned by the mother's work situation during confinement and having to help other people outside the home.

Keywords: University students; COVID-19; Lockdown; Anxiety; Empathy.

*Contacto: rlozano@unizar.es

ISSN: 2254-3139

www.rinace.net/riejs/

revistas.uam.es/riejs
Recibido: $\quad 15$ de junio 2020

$1^{\text {a }}$ Evaluación: 13 de septiembre 2020

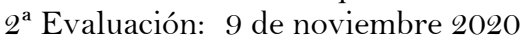

Aceptado: 16 de noviembre 2020 


\section{Introducción}

La excepcionalidad de la pandemia por Covid-19, ha supuesto un reto para las organizaciones educativas, siendo necesaria su adecuación tanto en materia docente como, en el caso de la educación superior, investigadora (Zhou et al., 2020). De esta forma, la universidad no sólo ha ejecutado y promovido la investigación sobre el COVID-19 y adaptado su docencia a las nuevas tecnologías (Fernandez y Shaw, 2020; Hiremath et al., 2020), sino que, además, se ha erigido como un canal de apoyo y colaboración hacia la comunidad y su estudiantado (Sina Financial News, 2020; Wang et al., 2020).

La aparición inicial de sintomatología de corte psicosocial en los estudiantes universitarios impulsó la investigación de la que forma parte el presente artículo. Ésta pretende arrojar luz sobre el estado de salud psicosocial de los jóvenes universitarios, con el último fin de comprender y responder de manera inclusiva e innovadora a las necesidades de este colectivo, que también ha sufrido las consecuencias del confinamiento, en términos de incremento de la ansiedad. Por otra parte, se explora también otra variable psicosocial relevante en este tipo de situaciones que, en positivo, puede promover actitudes prosociales que sirvan para superar las antedichas situaciones de angustia. Pare ello se propone un estudio cuantitativo basado en modelos de ecuaciones estructurales con una muestra de $\mathrm{n}=124$ universitarios, que respondieron a una encuesta enviada durante el período de confinamiento. En el SEM se introducen variables exógenas relacionadas con aspectos sociopersonales y, como variables endógenas, las medias de las escalas de ansiedad y empatía, obteniéndose un modelo final óptimo. La selección de variables deriva de la revisión de la literatura realizada de manera previa a la construcción del modelo, como presentamos a continuación.

\section{Revisión de la Literatura}

La irrupción de la pandemia por Covid-19 ha trastocado la vida cotidiana de la población. Concretamente en España ha sido necesario la implementación de fuertes medidas de confinamiento para evitar su propagación (Real Decreto 463/2020). El cierre de universidades presenciales ha supuesto la adaptación de la educación universitaria, que ha favorecido a la enseñanza online, hacia un modelo de liderazgo participativo y colaborativo (Fernandez y Shaw, 2020) así como a un seguimiento personalizado basado en la innovación educativa y el apoyo psicológico (Wang, Ng y Brook, 2020).

La Covid-19 no afecta por igual a todos los grupos de edad tanto en los síntomas fisiológicos (Mustafa y Selim, 2020) como en los psicológicos (World Health Organization, 2020). Los adultos jóvenes, en los que se encuadra la mayor parte de los estudiantes universitarios, son el grupo de edad que expresa un mayor malestar psicológico derivado, tanto del aislamiento como de la incertidumbre laboral y económica (Kilmer y Gil Rivas, 2010; Moghanibashi-Mansourieh, 2020; Wang y Tang, 2020), como del consumo continuo de información (Gao et al., 2020; Moghanibashi-Mansourieh, 2020), siendo especialmente vulnerables el estudiantado ante la denominada "infodemic" (Gao et al., 2020). Si bien, dentro de esta población se evidencian importantes diferencias de sexo, siendo las mujeres quienes presentan un mayor nivel de estrés psicosocial, ansiedad y depresión (Ahmed et al., 2020; Moghanibashi-Mansourieh, 2020; Wang y Tang, 2020). Hay que señalar que durante el confinamiento se ha perdido el contacto de tipo presencial con amistades lo que supone una red de apoyo social importante (Wind, Fordham y 
Komproe, 2011). El aumento de malestar psicológico en la población ha sido estudiado en pandemias anteriores; así Taylor y otros (2008) argumentan que las personas más jóvenes y con menos estudios son un grupo de riesgo en desarrollar estrés. Por otro lado, Everts (2013) defiende que la información proporcionada por los medios de comunicación, durante una crisis sanitaria, afectan al bienestar mental de la población. Además, Roy et al., (2020) tras realizar una comparativa entre varias pandemias concluyen que la extensión de la ansiedad a grandes grupos de población hace que las personas adopten hábitos no saludables de acuerdo con los rumores o fake nerws. Por lo tanto, es evidente que el contexto influye en los estados de ansiedad derivados del aislamiento social sobre todo (Lock y Gordon, 2012), pero también de lo relacionado con la excepcionalidad en la educación (Kilmer y Gil-Rivas, 2010) o la exposición a la información de los medios de comunicación de carácter catastrofista (Lau et al., 2010). A ello se debe añadir que ya en circunstancias habituales el uso excesivo de los mass media correlaciona con la ansiedad (Cerniglia et al., 2017; Mamun y Griffiths, 2019), siendo la edad una variable de vulnerabilidad (Andreassen et al., 2012; García-Domingo, Aranda y Fuertes, 2017).

No obstante, esta situación de aislamiento no solo se relaciona con la ansiedad, sino que la empatía es otra de las variables a considerar. La empatía es definida por Haut y otros (2019) como la capacidad que la persona tiene para comprender y compartir las emociones de los demás en un contexto relacional y prosocial. Durante la etapa de confinamiento ha sido un elemento primordial en la convivencia dentro del hogar. Algunos investigadores señalan que en situaciones de crisis los niveles de empatía aumentan y se manifiestan en un apoyo mayor entre los miembros convivientes (Siedlecki et al., 2014), otros argumentan que el efecto se exterioriza en aquellos que poseen mayor compromiso de cooperación (Glassman, 2000). Por otro lado, si el hecho de ser mujer incrementa los niveles de ansiedad, en la actual situación, también se relaciona con un aumento de empatía (Howard et al., 2018) que se manifiesta a la hora de manejar las distintas situaciones dentro del hogar con un espíritu cooperativo ante las adversidades (Howard et al., 2018; Kim y Zakour, 2017; Zakour y Gillespie, 2012). En definitiva, la empatía ayuda a generar un ambiente de bienestar psicológico en la convivencia familiar y ejerce un efecto positivo tanto en la persona que la da como en la que la recibe (Siedlecki et al., 2014; Vargas Murga, 2014; Welton-Michell, James, y Awale, 2016). Por otra parte, existen factores como el cuidado de familiares enfermos o dependientes que, aunque influyen positivamente en la empatía también introduce estresores que aumentan la ansiedad (López-Jiménez et al., 2011; Soriano-Fuentes, De la Torre-Rodríguez y Soriano-Fuentes, 2003).

Partiendo de estas premisas, el objetivo del presente estudio es investigar el nivel de ansiedad y de empatía que los estudiantes universitarios presentan como consecuencia de este confinamiento obligatorio y del cambio de estrategias a la hora de cursar las diferentes materias de sus carreras universitarias. También interesa el estudio de la influencia que distintos factores socio-demográficos puedan tener en estas variables, tales como el tipo de hogar, nivel de estudios de los padres, uso de las tecnologías de la información y la comunicación, etc. así como analizar las posibles diferencias en función del sexo. El interés de las instituciones educativas ha ido mayoritariamente dirigido al estudio relacionado con la población infantil y con su estado emocional como población considerada más vulnerable. No obstante, el alumnado universitario ha recibido menos atención en cuanto al estado de su salud mental y por ello el presente trabajo investiga las consecuencias que el confinamiento y el cambio de metodología de estudio ha podido afectar a su bienestar emocional. 


\section{Método}

La presente investigación fue llevada a cabo entre marzo y abril de 2020, durante el período de confinamiento del estado de alarma derivado de la crisis sanitaria del COVID19. A continuación, se detallan los aspectos metodológicos referidos a los participantes del estudio, el procedimiento seguido, incluyendo los estándares éticos que cumple la investigación, los instrumentos empleados para la recogida de datos y el análisis estadístico llevado a cabo.

\section{Participantes}

Los participantes de la investigación se seleccionaron mediante un muestreo no probabilístico por conveniencia y bola de nieve en el territorio español, pero con respuesta mayoritaria de estudiantes procedentes de la Comunidad Autónoma de Aragón (España), resultando un tamaño muestral total de $\mathrm{n}=124$. Aunque los participantes del estudio fueron familias, estudiantes y docentes, en el presente artículo nos referiremos únicamente a las respuestas de los estudiantes. En cuanto a la caracterización de la muestra, la edad media de los participantes se sitúa en 22,7 ( $\mathrm{DE}=7,8$ ). Las mujeres componían el segmento mayoritario $(84,7 \%)$ dada su mayor presencia en los grados de magisterio y máster de profesorado, estudios de los cuales procedían la mayoría de las participantes. El tipo de muestreo fue por conveniencia y bola de nieve, dadas las circunstancias de confinamiento en las que nos encontrábamos durante la realización del estudio. Tanto la situación, como este tipo de muestreo ha provocado que la muestra no sea muy amplia y exista una sobrerrepresentación de estudiantes del área de Educación, dado que los investigadores tenían un acceso más directo a ellos. Aunque esta presencia mayoritaria de estudiantes de Educación y mujeres puede provocar cierto sesgo en la muestra, como se reportará en la discusión, los resultados estaban alineados con la literatura científica base del estudio.

\section{Procedimiento}

Para la realización de este trabajo, una vez definidos los objetivos y metodología del mismo, se tramitó su aprobación por parte de la Comisión de Ética de la Universidad de Zaragoza actuando bajo las directrices del Comité de Ética de la Comunidad Autónoma de Aragón (CEICA). Tras dicha aprobación en marzo de 2020, en una primera fase, se lanzó un cuestionario de consentimiento informado a familias, docentes y estudiantes en redes sociales de confianza relacionadas con el ámbito educativo: colegios, institutos y universidades, incluyendo la información relativa a la naturaleza y objetivo de la investigación, su carácter anónimo, un formulario de contacto y la aceptación de ser incluidos en el estudio. En una segunda fase, se contactó con las personas que habían manifestado su voluntad de participación en el consentimiento informado garantizando la confidencialidad y el anonimato que respetaba los procedimientos éticos de la Declaración de Helsinki (World Medical Association, 2001). La tercera fase consistió en el envío de los cuestionarios que debían rellenar en formato online (Google Drive), sin incluir datos personales. Las respuestas fueron grabadas en una base de almacenamiento de datos digital para posteriormente ser exportadas con el único fin de investigación científica. Por último, de los datos totales se seleccionó el segmento de respuestas correspondiente a los estudiantes universitarios que se utilizó para caracterizarlos. Este proceso fue realizado al haber transcurrido veinte días de confinamiento, con la finalidad de asegurar el efecto de dicha medida. 


\section{Instrumentos}

Para medir el status socioeducativo y los datos demográficos se utilizó el Cuestionario de datos demográficos y socioeducativos ad hoc, de elaboración propia. Las preguntas de este cuestionario versaban sobre el tipo de estructura familiar, número de hermanos, tipo de hogar, nivel educativo, profesión y situación laboral de los entrevistados y sus progenitores, tiempo dedicado a tareas del hogar, tareas escolares, ejercicio o consumo de TV e Internet, etc. Consta de 40 ítems y fue evaluado mediante panel de expertos alcanzando un nivel de acuerdo casi perfecto $(k>0,81)$ siguiendo las indicaciones de Cohen (2013).

Para medir el nivel de ansiedad se utilizó el Inventario de ansiedad de Beck (BAI) en su adaptación española realizada por Sanz, García-Vera y otros (2005). Está diseñado para una recolección de información en formato autoinforme que permite medir el grado de ansiedad y los aspectos o síntomas menos relacionados con depresión. En concreto, mide la ansiedad derivada de trastornos de angustia, pánico y ansiedad generalizada siguiendo lo establecido por los criterios descritos en el DSM-III-R. Este cuestionario consta de 21 ítems cuyas respuestas bareman de 0 a 3 , siendo 0 en absoluto y 3 , severamente. El índice de fiabilidad de la escala en nuestra muestra fue muy óptimo, con un coeficiente de,92 en el alfa de Cronbach.

Para medir el nivel de empatía se utilizó la Escala de empatía cognitiva EQ Empaty Quotiente en su adaptación a la versión española (Redondo y Herrero-Fernández 2018). El cociente de empatía informa sobre la facilidad y disposición del individuo a captar y comprender los sentimientos de otras personas y como estos se ven afectados por los sentimientos de los demás. En este caso, el estudio se centró en la empatía de tipo cognitivo por lo que se utilizó únicamente esta sub-escala. Constó de 7 ítems, valorados ente 0 y 3 , siendo 0 en absoluto y 3 , severamente. El valor del alfa de Cronbach para esta subescala fue de 0,81 .

\section{Análisis}

Los análisis estadísticos se llevaron a cabo utilizando el software estadístico IBM SPSS Statistics para Windows. En primer lugar, se realizó un análisis descriptivo de las respuestas informadas de carácter socio-personal para caracterizar la muestra. Seguidamente, se exploraron las diferencias entre los niveles de empatía y ansiedad mediante el análisis de la varianza (ANOVA). Además, para establecer la relación entre los factores socioeducativos y los factores psicológicos de ansiedad y empatía cognitiva se llevó a cabo un análisis de correlación de Pearson, teniéndose en cuenta para el presente estudio solo aquellas relaciones que resultaran significativas $(p<0,05)$. Finalmente, teniendo en cuenta estas variables se diseñó un modelo de ecuaciones estructurales (SEM) (modelo 1) que no resultó totalmente óptimo, por lo que se eliminaron del modelo aquellas variables que no resultaron significativas, y se testó un segundo modelo (modelo 2) que cumplía con todos los criterios de los indicadores de bondad de ajuste.

La elección de la técnica SEM viene dada por la posibilidad de emplear en un mismo modelo varias variables independientes y dependientes, considerándose una ventaja por encima del análisis tradicional mediante regresiones lineales, además de la mayor fiabilidad de sus indicadores de bondad de ajuste (Byrne, 2010). Por otra parte, siguiendo a esta misma autora (Byrne, 2010, p. 5), otra de las ventajas fundamentales de este tipo de análisis es que permite incluir en un mismo modelo distintos tipos de variables exógenas 
o independientes (dicotómicas, nominales, ordinales, continuas, etc.), lo cual nos permitía construir un modelo que tuviese en cuenta las variables empleadas en nuestro cuestionario. No obstante, dado el tamaño de la muestra, el modelo solo soportó variables observadas y no latentes. La muestra fue suficiente para el modelo, dado que, siguiendo a Catena y otros (2003), una muestra adecuada sería de 8 casos por el total de variables observadas, si bien, Hair y otros (2014) señalan que 15 casos por indicador o variable observada da como resultado una muestra apropiada que incluso permite minimizar problemas de normalidad multivariable. Dado que nuestro modelo 1 tenía 11 variables observadas, la muestra debía ser de mínimo $\mathrm{n}=88$ para ser adecuada, si bien nuestro segundo modelo, al reducirse a 7 variables observadas, cumplía el criterio de Hair y otros (2014) de 15 casos por variable (mínimo de 105 casos), superándose con el tamaño de $\mathrm{n}=$ 124. Prueba de ello fue el cumplimiento de todos los criterios de mondad de ajuste del modelo 2 .

\section{Resultados}

Los resultados del análisis descriptivo de carácter socio-personal muestran que un 46,8\% vivía con los dos progenitores. El 35,5\% afirmaba convivir con una persona considerada "de riesgo" (personas mayores, personal sanitario o de seguridad, etc.) y un $22,7 \%$ remarcaba que esa persona de riesgo precisaba de asistencia. El nivel de estudios predominante en los progenitores son los secundarios, tanto en el caso del padre (51,3\%) como en el caso de la madre $(39,5 \%)$. En cuanto a la situación laboral durante el confinamiento, el 23,5\% de las madres mantenían el trabajo presencial, mientras que el 21,8\% estaba en una situación de Expediente de Regulación Temporal de Empleo (ERTE) y el 16,8\% teletrabajaba. En el caso de los padres, esos porcentajes eran del 25,9\%, 27,7\% y $9,8 \%$ respectivamente. En cuanto a las actividades durante el confinamiento, el 42,7\% afirmaba dedicar de 4 a 6 horas a las tareas universitarias y un $37,1 \%$ realizaba actividad física cada día, si bien un tercio de los encuestados decía que su consumo de TV se había visto incrementado de forma moderada o severa, y un $75,8 \%$ en el caso del consumo de Internet.

En cuanto a los niveles de ansiedad de los entrevistados se situaban en 18,1 (DE 11,4) sobre 45,0 y los de empatía en 13,5 (DE 4,2) sobre 21,0. Teniendo en cuenta solo las variables socio-personales que en el estudio preliminar resultaron significativas, los resultados del test ANOVA se detalla en el cuadro 1. En él, podemos apreciar que los niveles de ansiedad varían significativamente si atendemos al sexo (19,2 en mujeres respecto al 10,0 en hombres); las personas con las que se convive en el hogar, siendo mucho más alta $(22,4)$ cuando se convive con personas mayores; la educación de la madre (más elevada en personas con una progenitora con menor nivel de estudios) y por el incremento del consumo de TV e Internet (23,4 y 19,9 respectivamente cuando dicho consumo ha aumentado severamente). Los niveles de empatía se ven incrementados cuando la madre no trabaja $(14,8)$, teletrabaja $(18,0)$, la han despedido $(19,5)$ o es ama de casa $(14,5)$, es decir, cuando su vinculación con su puesto de trabajo físico es menor, y cuando el padre no trabaja $(15,0)$. Paradójicamente, el estudiantado que afirman ayudar a familiares fuera de su domicilio presenta niveles menores de empatía (12,3 frente a 14,3). 
Cuadro 1. Niveles de ansiedad y empatía por variables socio-personales. Media, desviación estándar y nivel de significatividad estadística

\begin{tabular}{|c|c|c|c|}
\hline VARIABLES & INDICADORES & ANSIEDAD & EMPATÍA \\
\hline \multirow[t]{2}{*}{ Sexo } & Mujer & $19,6(11,1)^{* * *}$ & $13,8(3,9)$ \\
\hline & Hombre & $10,0(9,4)$ & $11,8(5,4)$ \\
\hline \multirow{3}{*}{ Hogar } & Vive solo & $6,0(8,5)$ & $12,5(7,8)$ \\
\hline & Vive con otros adultos (padres, pareja, amigos) & $17,3(11,1)$ & $13,9(4,2)$ \\
\hline & Vive con alguna persona mayor & $22,4(11,5)^{*}$ & $12,3(4,0)$ \\
\hline \multirow{4}{*}{ EdMadre } & Sin estudios & $26,8(9,6)$ & $15,0(2,4)$ \\
\hline & Primaria /EGB & $16,7(11,6)$ & $13,6(4,1)$ \\
\hline & Estudios secundarios & $21,0(10,6)^{*}$ & $13,5(4,6)$ \\
\hline & Estudios universitarios & $14,5(11,7)$ & $13,2(4,1)$ \\
\hline \multirow[t]{2}{*}{ OcMadre } & Trabaja & $17,1(11,5)$ & $12,9(4,1)$ \\
\hline & No trabaja & $20,3(10,9)$ & $14,8(4,1)^{*}$ \\
\hline \multirow[t]{2}{*}{ OcPadre } & Trabaja & $17,8(11,5)$ & $13,1(4,2)$ \\
\hline & No Trabaja & $19,6(11,0)$ & $15,0(4,1)^{*}$ \\
\hline \multirow[t]{6}{*}{ SLabMadre } & Acude al puesto de trabajo & $16,0(12,0)$ & $12,5(4,0)$ \\
\hline & ERTE & $18,5(11,5)$ & $12,6(4,3)$ \\
\hline & Teletrabaja (online) & $15,6(11,2)$ & $13,0(3,5)$ \\
\hline & Teletrabaja (otros) & $22,0(12,2)$ & $18,0(2,6)$ \\
\hline & Despedida & $13,0(7,1)$ & $19,5(2,1)^{*}$ \\
\hline & Ama de casa & $20,8(11,0)$ & $14,5(4,3)$ \\
\hline \multirow{2}{*}{ AyudaFH } & No & $17,6(11,2)$ & $14,3(4,2)^{*}$ \\
\hline & Sí & $19,0(11,7)$ & $12,3(3,9)$ \\
\hline \multirow[t]{4}{*}{ TV } & No & $15,0(11,6)$ & $13,0(4,1)$ \\
\hline & Levemente & $18,8(10,8)$ & $13,5(4,4)$ \\
\hline & Moderadamente & $18,6(11,5)$ & $13,9(4,1)$ \\
\hline & Severamente & $23,4(11,6)^{*}$ & $13,8(4,1)$ \\
\hline \multirow[t]{4}{*}{ Internet } & No & $8,0(7,5)$ & $15,3(2,5)$ \\
\hline & Levemente & $15,1(10,9)$ & $12,7(4,7)$ \\
\hline & Moderadamente & $18,9(11,8)$ & $13,6(4,1)$ \\
\hline & Severamente & $19,9(10,8)^{*}$ & $13,7(4,1)$ \\
\hline
\end{tabular}

Nota: $* \mathrm{p}<0,05 ; * * \mathrm{p}<0,01$.

Fuente: Elaboración propia.

Antes de testar el modelo estructural se procedió a evaluar la correlación entre las variables que se iban a emplear en el modelo y que habían resultado significativas en el test ANOVA. Como se puede apreciar en el cuadro 2, las relaciones que habían resultado significativas en el test anterior también emergen en el análisis de correlaciones. En relación a la ansiedad, la variable sexo presenta la mayor significatividad $\left(r=-0,306^{* * * *}\right)$, es decir, las mujeres presentan niveles de ansiedad superiores a los varones. También correlaciona significativamente con el tipo de hogar $\left(r=0,220^{*}\right)$, con el nivel educativo de la madre $\left(r=0,207^{*}\right)$ y con el consumo de internet y de TV $\left(r=0,190^{*}, r=0,187^{*}\right)$. En el caso de la empatía es la situación laboral de la madre ( $\left.r=0,241^{* *}\right)$, es decir, los hijos de aquellas que pasan más tiempo en casa poseen mayor empatía. Del mismo modo presentan una relación significativa con la ayuda fuera del hogar $\left(\mathrm{r}=-0,229^{*}\right)$ y con la ocupación del padre $\left(\mathrm{r}=0,183^{*}\right)$. Existe también una correlación significativa entre los niveles de ansiedad y empatía $\left(\mathrm{r}=0,286^{* * * *}\right)$. Además, surgen otras relaciones interesantes para el diseño posterior del modelo SEM, como las relaciones entre sexo y nivel educativo de la madre $\left(r=-0,180^{*}\right)$, sexo y consumo de Internet $\left(r=-0,211^{*}\right)$, nivel educativo de la madre y ocupación de la madre $\left(r=0,209^{*}\right)$, nivel educativo de la madre y ayuda fuera del hogar $\left(r=-0,229^{*}\right)$ y ocupación de la madre y situación laboral de la madre $\left(\mathrm{r}=0,661^{* * * *}\right)$. 
Cuadro 2. Coeficientes de correlación de Pearson y niveles de significatividad entre variables

\begin{tabular}{|c|c|c|c|c|c|c|c|c|c|c|}
\hline & 1 & 2 & 3 & 4 & 5 & 6 & 7 & 8 & 9 & 10 \\
\hline \multicolumn{11}{|l|}{ 1. Sexo } \\
\hline 2. Hogar & $-0,04$ & & & & & & & & & \\
\hline 3. EdM & $-0,18^{*}$ & 0,17 & & & & & & & & \\
\hline 4. OcM & $-0,05$ & 0,05 & $0,21^{*}$ & & & & & & & \\
\hline 5. OcP & 0,07 & $-0,13$ & 0,14 & $0,21^{*}$ & & & & & & \\
\hline 6. SLabM & $-0,03$ & $-0,04$ & 0,10 & $0,66^{* * * *}$ & $0,19^{*}$ & & & & & \\
\hline 7. AyuFH & $-0,06$ & 0,10 & $-0,23^{*}$ & $-0,16$ & $-0,15$ & 0,01 & & & & \\
\hline 8. TV & $-0,07$ & $-0,09$ & 0,10 & $-0,08$ & 0,00 & 0,02 & $-0,01$ & & & \\
\hline 9. Internet & $-0,22^{*}$ & $-0,12$ & $-0,03$ & $-0,01$ & $-0,08$ & 0,10 & 0,11 & $0,29^{* * *}$ & & \\
\hline 10. Ansied & $-0,31^{* * *}$ &, $22^{*}$ & $0,21^{*}$ & 0,13 & 0,07 & 0,16 & 0,06 & $0,10^{*}$ & $0,19^{*}$ & \\
\hline 11. Empat & $-0,17$ & $-0,14$ & 0,06 & $0,20^{*}$ & $0,183^{*}$ & $0,24^{* * *}$ & $-0,23^{*}$ & 0,08 & 0,05 & $0,29^{* * * *}$ \\
\hline
\end{tabular}

A continuación, se procedió a testar el modelo hipotético (figura 1) derivado de la exploración de los datos previos. Este modelo 1 explica el 13,9\% de la varianza en empatía y el $17,8 \%$ de la varianza en los niveles de ansiedad. Sin embargo, no todas las relaciones resultaron óptimas, los pesos que resultaron significativos fueron la influencia del sexo ( $\beta$ $\left.=-0,191^{* * *}\right)$, el tipo de hogar $\left(\beta=0,316^{* * *}\right)$ y el incremento en el consumo de TV ( $\beta=$ $\left.0,142^{*}\right)$ en la ansiedad; y de la ayuda fuera del hogar $\left(\beta=-0,264^{* * *}\right)$ y la situación laboral de la madre $\left(\beta=0,242^{* * *}\right)$ en la empatía (cuadro 3$)$.

Debido a ello se procedió a testar un segundo modelo prescindiendo de las variables exógenas cuyo peso en las variables endógenas no había resultado significativo en el modelo 1. El modelo 2 explicaba el $7,9 \%$ de la varianza en la empatía y el 16,2\% en ansiedad. En él, la ansiedad se ve influida por el sexo ( $\left(=-0,191^{* *}\right)$, constatándose que el hecho de ser mujer está relacionado con mayores niveles de ansiedad, el tipo de hogar ( $B$ $=0,312^{* * *}$ ), por lo que convivir con personas mayores (personas de riesgo) incrementa notablemente los niveles de ansiedad, y el incremento del consumo de TV ( $\left(=0,164^{*}\right)$. La empatía, en cambio, se ve incrementada cuando la situación laboral de la madre se desvincula de ir al lugar físico de trabajo $\left(\beta=0,175^{*}\right)$ y cuando no se tiene que ir fuera del hogar a ayudar a alguna persona de riesgo $\left(B=-0,221^{*} *\right)$ (cuadro 4$)$. Este segundo modelo presenta unos indicadores de bondad de ajuste más óptimos que el modelo 1 , tal y como puede apreciarse en el cuadro 5.

Cuadro 3. Modelo estructural 1 de variables socio-personales que influyen en los niveles de empatía y ansiedad de los estudiantes durante el período del confinamiento. Coeficientes estandarizados y no estandarizados

\begin{tabular}{lllccc}
\hline & & $\begin{array}{c}\text { CoEF. NO } \\
\text { ESTANDARIZADOS }\end{array}$ & S.E. & C.R. & $\begin{array}{c}\text { CoEF. } \\
\text { ESTANDARIZADOS }\end{array}$ \\
\hline Ansiedad $<--$ & Sexo & $-5,894$ & 1,679 & $-3,510$ & $-0,191^{* * *}$ \\
Ansiedad $<--$ & Hogar & 8,141 & 1,836 & 4,433 & $0,316^{* * *}$ \\
Ansiedad $<--$ & EdMadre & 0,568 & 0,655 & 0,867 & 0,065 \\
Ansiedad $<--$ & TV & 1,561 & 0,851 & 1,834 & $0,142^{*}$ \\
Ansiedad $<--$ & Internet & 0,866 & 0,987 & 0,877 & 0,063 \\
Empatía $<--$ & OcMadre & $-0,319$ & 1,056 &,- 302 & $-0,036$ \\
Empatía $<--$ & OcPadre & 1,105 & 0,739 & 1,495 & 0,097 \\
Empatía $<--$ & AyudaFH & $-2,162$ & 0,541 & $-3,996$ & $-0,264^{* * *}$ \\
Empatía $<--$ & SLabMadre & 0,530 & 0,235 & 2,260 & $0,272^{*}$ \\
\hline
\end{tabular}

Nota: *p $<0,05$; ** $p<0,01,{ }^{*} * * *$ p $<0,001 .<--$ Efecto de la variable exógena sobre la variable endógena en el modelo SEM.

Fuente: Elaboración propia. 


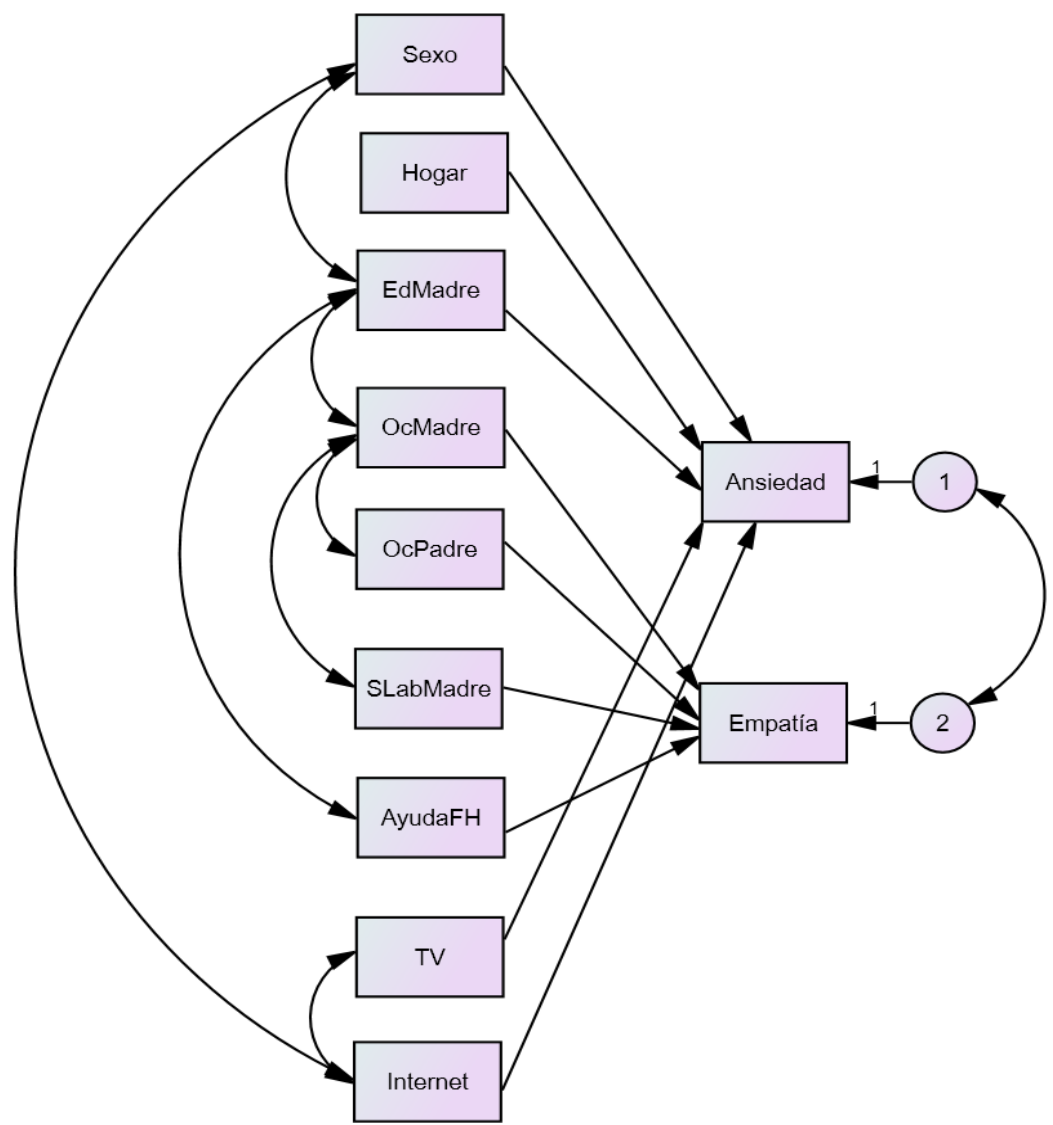

Figura 1. Representación gráfica del modelo 1 Fuente: Elaboración propia.

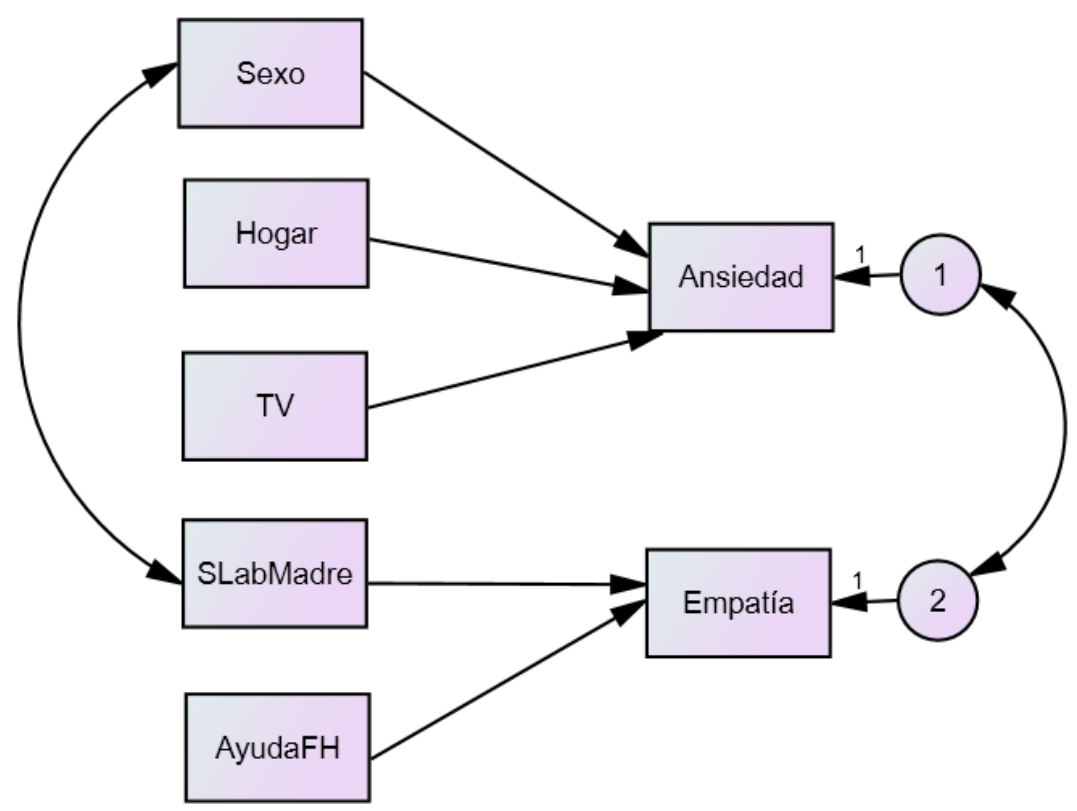

Figura 2. Representación gráfica del modelo 2 Fuente: Elaboración propia. 
Cuatro 4. Modelo estructural 2 de variables socio-personales que influyen en los niveles de empatía y ansiedad de los estudiantes durante el período del confinamiento. Coeficientes estandarizados y no estandarizados

\begin{tabular}{lllcccc}
\hline & & & COEF. NO ESTAND. & S.E. & C.R. & COEF. ESTAND. \\
\hline Ansiedad & $<--$ & Sexo & $-6,204$ & 2,004 & $-3,095$ & $-0,193^{* *}$ \\
Ansiedad & $<--$ & Hogar & 8,078 & 1,994 & 4,051 & $0,312^{* * *}$ \\
Ansiedad & $<--$ & TV & 1,905 & 0,904 & 2,108 & $0,164^{*}$ \\
Empatía & $<--$ & AyudaFH & $-1,739$ & 0,602 & $-2,890$ & $-0,221^{* *}$ \\
Empatía & $<--$ & SLabMadre & 0,330 & 0,157 & 2,096 & $0,175^{*}$ \\
\hline
\end{tabular}

Nota: ${ }^{*} \mathrm{p}<0,05 ;$ ** $\mathrm{p}<0,01$, **** $^{*} \mathrm{p}<0,001 .<-$ Efecto de la variable exógena sobre la variable endógena en el modelo SEM.

Fuente: Elaboración propia.

Cuadro 5. Comparativa de indicadores de bondad de ajuste entre el modelo 1 y 2

\begin{tabular}{lcc}
\hline \multicolumn{1}{c}{ INDICADOR } & MODELO 1 & MODELO 2 \\
\hline GFI & 0,98 & 0,99 \\
CFI & 0,92 & 0,99 \\
TLI & 0,89 & 0,99 \\
RMSEA & 0,08 & 0,01 \\
Chi2/GL & 1,72 & 1,01 \\
\hline
\end{tabular}

Fuente: Elaboración propia.

\section{Discusión}

De inicio debemos atender a una serie de limitaciones a considerar en el presente estudio. Por un lado, hubiese sido constructivo poder haber contado con la participación de una muestra más diversificada de estudiantes universitarios (con mayor equilibrio entre sexos, de distintas áreas, o matriculados en grados online o semipresenciales, entre otros). Como se ha especificado en el apartado de "Participantes" el tipo de muestreo por conveniencia, derivado de la situación y la consecuente limitación de acceso a un mayor número de estudiantes, ha provocado que nuestra muestra pueda considerarse algo sesgada, debido a la sobrerrepresentación de estudiantes del área de Educación y de mujeres. Esta limitación ha podido provocar las diferencias significativas a favor del segmento de las estudiantes. Unido a ello, el tamaño muestral, aunque suficiente para la realización de un análisis SEM, insta a tomar con cautela los resultados a la hora de inferirlos. Sin embargo, aunque estas condiciones han podido influir en un porcentaje de la varianza explicada relativamente bajo, tanto el carácter óptimo de los indicadores de bondad de ajuste del modelo, como el alineamiento de los resultados con estudios previos (por ejemplo, el incremento de los niveles de ansiedad en estas situaciones asociados en mayor medida a las mujeres), nos lleva a considerar su aceptabilidad. Otra limitación con la cuenta la presente investigación es la ausencia de una medición previa al confinamiento. Para controlar esta situación se decidió retrasar las pruebas hasta el veinteavo día de confinamiento para asegurar el efecto de dicha medida.

Como futuras líneas de investigación con estudiantes universitarios sería muy relevante replicar el estudio en situaciones distintas a las del contexto de confinamiento, y tener en cuenta una muestra con un mayor equilibrio en cuanto a hombres y mujeres. Por otro lado, sería interesante estudiar el papel de la variable infodemic (World Health Organization, 2020), es decir, la infoxicación que aumenta la incertidumbre y el miedo sobre el Covid-19. Igualmente, consideramos necesario implementar prácticas de éxito en 
materia de tratamiento de información como parte de las competencias de alfabetización mediática, y método científico, propias de los grados universitarios. En pandemias previas, se ha estudiado la transcendencia de esta variable en la población joven (Everts, 2013; Roy et al., 2020; Taylor et al., 2008). Es necesario determinar el papel que pueden jugar las universidades en la mejor adquisición de competencias de alfabetización mediática y tratamiento de información de tipo seudocientífico. Es por ello determinante, desarrollar programas de intervención en innovación docente que permitan tanto evaluar cómo mejorar el nivel de competencia

Las pandemias no sólo refieren a síntomas fisiológicos sino también a psicosociales (World Health Organization, 2020) enmarcando la salud desde el modelo biopsicosocial propuesto por la Organización Mundial de la Salud. La pandemia por Covid-19 ha afectado de manera considerable a los estudiantes universitarios, quienes además de continuar con su formación han tenido que sobrellevar su malestar psicológico, la incertidumbre laboral y económica, así como la ayuda al cuidado de familiares enfermos.

Los resultados del presente estudio evidencian que el sexo del entrevistado, el tipo de hogar (personas con las que convive) y el aumento en el consumo de TV influyen en los niveles de ansiedad en esta situación de confinamiento. Estos resultados concuerdan con estudios previos sobre la pandemia por covid-19, quienes señalan como la población joven femenina es uno de los grupos más afectados (Ahmed et al., 2020; MoghanibashiMansourieh, 2020; Wang y Tang, 2020). Por otro lado, el tipo o estructura familiar también presenta un aumento de la ansiedad en los universitarios, mostrando menor nivel de ansiedad cuando se convive en un hogar estructurado y sobre todo cuando está formado por un número mayor de miembros. La familia favorece una buena salud y calidad de vida, pero en algunos casos también genera conflictos que pueden desembocar en problemas de salud mental (Vargas Murga, 2014). Hay que tener en cuenta que estar sometido a situaciones emergentes o a la presencia de familiares enfermos o incapacitados añade elementos estresantes en las relaciones familiares (López-Jiménez et al., 2011). Las investigaciones anteriores no son concluyentes, por ejemplo, Soriano-Fuentes, De la Torre-Rodríguez y Soriano-Fuentes (2003) no encontraron relación significativa entre el tipo de familia y la presencia de ansiedad, depresión, etc. En cambio, Montalbán Sánchez (1998) halló que el tamaño de la familia se relacionaba con la presencia de ansiedad, pero no así con el tipo de familia. Por otro lado, Pérez Milena (2007) realizó su estudio en alumnos de secundaria y concluyó que la estructura familiar no influía en la aparición de trastornos mentales y matizaba que factores demográficos y los recursos disponibles presentaban mayor relación. Por lo tanto, el funcionamiento familiar más que el tipo de familia tendría alguna relación con los niveles de ansiedad en los componentes de la misma. Igualmente, los medios de comunicación parecen jugar un factor determinante reforzando la sintomatología ansiosa, de tal forma que a mayor consumo mayor ansiedad. Estos resultados son coherentes con estudios con otras poblaciones tanto durante la pandemia por Covid-19 (Gao et al., 2020; Moghanibashi-Mansourieh, 2020) como en pandemias previas (Everts, 2013; Taylor et al. 2008). Además, en ambientes normotípicos se debe tener presente que la edad es un factor de riesgo en el uso excesivo de los mass media (Andreassen et al., 2012; García-Domingo, Aranda y Fuertes, 2017), teniendo una elevada correlación con la ansiedad (Cerniglia et al., 2017; Mamun y Griffiths, 2019).

Los resultados relativos a la empatía ponen de manifiesto que no prestar ayuda fuera del hogar a personas dependientes, y la mayor presencia de horas de la madre en el hogar elevan los niveles de empatía. Hay que destacar que, la convivencia con personas enfermas 
en el hogar, ha supuesto para muchos estudiantes la adquisición del rol de cuidador, cuya actividad principal se fundamenta en atender a las necesidades de la persona dependiente (Dwyer et al., 1994). Esta situación ha derivado en un notable crecimiento de la ansiedad a la vez que ha disminuido su empatía cognitiva. A priori, estos datos son opuestos a los referenciados por autores como Kim y Zakour (2017) o Zakour y Gillespie (2012) quienes defienden que en crisis humanitarias o catástrofes naturales el ser humano es eminentemente social y genera redes sociales de apoyo. Si bien, se debe atender a la particularidad de esta crisis. Es posible, que la adquisición del rol de cuidador, unido, a sus obligaciones como estudiantes $\mathrm{y}$, al estado de confinamiento, haya repercutido en el estado emocional de los estudiantes universitarios, de tal forma que presentan menor empatía cognitiva y mayor ansiedad (Kilmer y Gil Rivas, 2010). Por otra parte, la empatía se incrementa cuando la actividad de la madre se desvincula del lugar físico de trabajo, permaneciendo mayor tiempo en el hogar. Es decir, se evidencia como la presencia ininterrumpida ha podido contribuir a una mejor comunicación, favoreciendo la escucha activa entre hijos (adultos jóvenes) y madres durante el confinamiento. Es decir, se ha producido un escenario para el cambio tras la gestión de esta crisis (Familia igualdad y bienestar social, 2020). Y es que el apoyo social percibido entre miembros de una entidad familiar se relaciona con mayor empatía (Siedlecki et al., 2014).

\section{Conclusiones y prospectiva de la investigación}

La presente investigación ha puesto de relieve cómo en una situación de emergencia, como la vivida, los universitarios se han visto afectados en sus niveles de ansiedad y empatía cognitiva, sobre todo en función del sexo, el entorno familiar, el trabajo de la progenitora y el uso de las tecnologías de la información y la comunicación. El sexo femenino se ha mostrado más vulnerable a la hora de afrontar esta nueva realidad por lo que ha presentado mayores niveles de ansiedad. La familia, pero sobre todo la figura materna ha sido un punto de anclaje para llevar lo mejor posible este modo de vida, en la que los jóvenes se han visto privados de las relaciones sociales presenciales con sus iguales

En cuanto futuras implementaciones de esta investigación, el tamaño muestral, aunque suficiente para la aplicación del modelo SEM, debería ampliarse en futuros estudios para lograr una mayor representatividad. La muestra, aunque ciertamente sesgada, dada las limitaciones de acceso a los participantes, con sobrerrepresentación de estudiantes de la región de Aragón, del área de Educación y mujeres, ha dado lugar a resultados lineados con la literatura previa, si bien se precisará en un futuro de una mayor diversificación. Las diferencias de los efectos teniendo en cuenta la variable "sexo", tanto en nuestra muestra como en estudios previos, exigen, por una parte, y como se ha especificado en las limitaciones, muestras equilibradas en relación a esta variable, y, una mayor profundización en la exploración de la causalidad de la mayor afección de los niveles de ansiedad en las mujeres. En coherencia, se ha planteado una nueva investigación que pretende estudiar las consecuencias psicosociales y laborales de esta pandemia profundizando en estas diferencias entre hombres y mujeres con una muestra representativa de toda la nación. 


\section{Referencias}

Ahmed, M. Z., Ahmed, O., Aibao, Z., Hanbin, S., Siyu, L. y Ahmad, A. (2020). Epidemic of COVID19 in China and associated psychological problems. Asian Journal of Psychiatry, 51, art 4. https://doi.org/10.1016/j.ajp.2020.102092

Andreassen, C. S., Torsheim, T., Brunborg, G. S. y Pallesen, S. (2012). Development of a Facebook addiction scale. Psychological Reports, $110(2)$, 501-517. https://doi.org/10.2466/02.09.18.PRo.110.2.501-517

Catena, A., Ramos, M. y Trujillo, H. (2003). Análisis multivariado: Un manual para investigadores. Biblioteca Nueva.

Cerniglia, L., Zoratto, F., Cimino, S., Laviola, G., Ammaniti, M. y Adriani, W. (2017). Internet addiction in adolescence: Neurobiological, psychosocial and clinical issues. Neuroscience $y$ Biobehavioral Reviews, 76, 174-184. https://doi.org/10.1016/j.neubiorev.2016.12.024

Cohen, J. (2013). Statistical power analysis for the behavioral sciences. Routledge. https://doi.org/10.4324/9780203771587

Dwyer, J. W., Lee, G. R. y Jankowski, T. B. (1994). Reciprocity, elder satisfaction, and caregiver stress and burden: The exchange of aid in the family caregiving relationship. Journal of Marriage and the Family, 56(1), 35-39. https://doi.org/10.2307/352699

Everts, J. (2013). Announcing swine flu and the interpretation of pandemic anxiety. Antipode, 45(4), 809-825. https://doi.org/10.1111/j.1467-8330.2012.01021.x

Familia igualdad y bienestar social. (2020). Guía para la convivencia familiar durante la crisis del COVID19. Ayuntamiento de Madrid.

Fernandez, A. A. y Shaw, G. P. (2020). Academic leadership in a time of crisis: The coronavirus and COVID-19. Journal of Leadership Studies, 14(1), 39-45.

https://doi.org/10.1002/jls.21684

Gao, J., Zheng, P., Jia, Y., Chen, H., Mao, Y., Chen, S., Wang, Y., Fu, H. y Dai, J. (2020). Mental health problems and social media exposure during COVID-19 outbreak. PLoS ONE, 15(4), 1-10. https://doi.org/10.1371/journal.pone.0231924

García-Domingo, M., Aranda, M. y Fuentes, V. M. (2017). Facebook use in university students: Exposure and reinforcement search. Procedia - Social and Behavioral Sciences, 237, 249-254. https://doi.org/10.1016/j.sbspro.2017.02.071

Glassman, M. (2000). Mutual aid theory and human development: Sociability as primary. Journal for the Theory of Social Behaviour, 30(4), 391-412. https://doi.org/10.1111/1468-5914.0013

Hair Jr., J., Black, W., Babin, B. y Anderson, R. (2014). Multivariate data analysis: Pearson new international edition. Pearson.

Haut, K. M., Dodell-Feder, D., Guty, E., Nahum, M. y Hooker, C. I. (2019). Change in objective measure of empathic accuracy following social cognitive training. Frontiers in Psychiatry, 10, 1-11. https://doi.org/10.3389/fpsyt.2019.00894

Hiremath, P., Suhas Kowshik, C. S., Manjunath, M. y Shettar, M. (2020). COVID 19: Impact of lock-down on mental health and tips to overcome. Asian Journal of Psychiatry, 51, 102088. https://doi.org/10.1016/j.ajp.2020.102088

Howard, A., Agllias, K., Bevis, M. y Blakemore, T. (2018). How social isolation affects disaster preparedness and response in Australia: Implications for social work. Australian Social Work, 71(4), 392-404. https://doi.org/10.1080/0312407X.2018.1487461 
Kilmer R. P. y Gil-Rivas V. (2010). Responding to the needs of children and families after a disaster: linkages between unmet needs and caregiver functioning. American Journal of Orthopsychiatry, 80(1),135-142. https://doi.org/10.1111/j.1939-0025.2010.01016.x

Kim, H. y Zakour, M. (2017). Disaster preparedness among older adults: Social support, community participation, and demographic characteristics. Journal of Social Service Research, 43(4), 498-509. https://doi.org/10.1080/01488376.2017.1321081

Lau, J. T., Yu, X., Zhang, J., Mark, W. W., Choi, K. C., Lui, W. W. y Chan, E. Y. (2010). Psychological distress among adolescents in Chengdu, Sichuan at 1 month after the 2008 Sichuan earthquake. Journal of Urban Health: Bulletin of the New York Academy of Medicine, 87(3), 504-523. https://doi.org/10.1007/s11524-010-9447-3

Lock, M. y Gordon, D. (2012). Biomedicine examined. Springer Science.

López-Jiménez, M.T., Barrera, M. I., Cortés, J. F. y Metzi, M. J. (2011). Funcionamiento familiar, creencias e inteligencia emocional en pacientes con trastorno obsesivo compulsivo y sus familiares. Salud Mental, 34, 111-120.

Mamun, M. A. y Griffiths, M. D. (2019). The association between facebook addiction and depression: A pilot survey study among Bangladeshi students. Psychiatry Research, 271, 628633. https://doi.org/10.1016/j.psychres.2018.12.039

Moghanibashi-Mansourieh, A. (2020). Assessing the anxiety level of Iranian general population during COVID-19 outbreak. Asian Journal of Psychiatry, 51, 102076. https://doi.org/10.1016/j.ajp.2020.102076

Montalbán Sánchez J. (1998). Relación entre ansiedad y dinámica familiar. Atención Primaria, 21, 29-34.

Mustafa, N. M. y Selim, L. A. (2020). Characterization of COVID-19 pandemic in pediatric age group: A systematic review and meta-analysis. Journal of Clinical Virology, 128(3), 104395. https://doi.org/10.1016/j.jcv.2020.104395

Navarro-Abal, Y., López-López, M. J., Climent-Rodríguez, J. A. y Gómez-Salgado, J. (2019). Burden, empathy, and resilience in dependent people caregivers. Gaceta Sanitaria, 33(3), 268-271. https://doi.org/10.1016/j.gaceta.2017.11.009

Pérez Milena A. (2007). Estructura y funcionalidad de la familia en la adolescencia: relación con el apoyo social, el consumo de tóxicos y el malestar psíquico. Atención Primaria, 39(2), 61-67. https://doi.org/10.1157/13098670

Redondo, I. y Herrero-Fernández, D. (2018). Adaptación del empathy quotient (EQ) en una muestra española. Terapia psicológica, 36(2), 81-89. https://doi.org/10.4067/So7 18-48082018000200081

Roy, D., Tripathy, S., Kar, S. K., Sharma, N., Verma, S. K. y Kaushal, V. (2020). Study of knowledge, attitude, anxiety yamp; perceived mental healthcare need in Indian population during COVID-19 pandemic. Asian Journal of Psychiatry, 51(10), 20-83. https://doi.org/10.1016/j.ajp.2020.102083

Sanz, J., García-Vera, M. P., Espinosa, R., Fortún, M. y Vázquez, C. (2005). Adaptación española del inventario para la depresión de Beck-II (BDI-II): 3. Propiedades psicométricas en pacientes con trastornos psicológicos. Clínica y Salud, 16, 121-142.

Siedlecki, K. L., Salthouse, T. A., Oishi, S. y Jeswani, S. (2014). The relationship between social support and subjective well-being across age. Social Indicators Research, 117(2), 561-576. https://doi.org/10.1007/s1 1205-013-0361-4 
Sina Financial News. (2020). Guarding the "fight" of the "native family": Huake alumni helped the Hubei to fight the epidemic, and supplies were sent directly to the front-line departments. http://finance.sina.com.cn/wm/2020-02-08/doc-iimxyqvz 1336454.shtml

Soriano-Fuentes, S., De la Torre-Rodríguez, R. y Soriano-Fuentes, L. (2003). Familia, trastornos mentales y ciclo vital familiar. Medicina de Familia, 2, 130- 135.

Taylor, M. R., Agho, K. E., Stevens, G. J. y Raphael, B. (2008). Factors influencing psychological distress during a disease epidemic: Data from Australia’s first outbreak of equine influenza. BMC Public Health, 8(1), 347. https://doi.org/10.1186/1471-2458-8-347

Vargas Murga, H. (2014). Tipo de familia y ansiedad y depresión. Revista Médica Herediana, 25(2), 57-59.

Vázquez-Sánchez, M. A., Aguilar-Trujillo, M. P., Estébanez-Carvajal, F. M., Casals-Vázquez, C., Casals-Sánchez, J. L. y Heras-Pérez, M. C. (2012). Influencia de los pensamientos disfuncionales en la sobrecarga de los cuidadores de personas dependientes. Enfermería Clínica, 22(1), 11-17. https://doi.org/10.1016/j.enfcli.2011.07.002

Wallace, C. L., Wladkowski, S. P., Gibson, A. y White, P. (2020). Grief during the COVID-19 pandemic: considerations for palliative care providers. Journal of Pain and Symptom Management, 4, 12-34. https://doi.org/10.1016/j.jpainsymman.2020.04.012

Wang, C. J., Ng, C. Y. y Brook, R. H. (2020). Response to COVID-19 in Taiwan: Big data analytics, new technology, and proactive testing. Journal of the American Medical Association, 323(14), 1341-1342. https://doi.org/10.1001/jama.2020.3151

Wang, Z. y Tang, K. (2020). Combating COVID-19: Health equity matters. Nature Medicine, 26(4), 458. https://doi.org/10.1038/s41591-020-0823-6

Welton-Mitchell, C., James, L. y Awale, R. (2016). Nepal 2015 earthquake: A rapid assessment of cultural, psychological and social factors with implications for recovery and disaster preparedness. International Journal of Mass Emergencies y Disasters, 34(3), 399-418.

Wind, T. R., Fordham, M. y H. Komproe, I. (2011). Social capital and post-disaster mental health. Global Health Action, 4(1), 1-10. https://doi.org/10.3402/gha.v4io.6351.

World Health Organization. (2020). Mental health and psychosocial considerations during the COVID19 outbreak. World Health Organization.

World Medical Association. (2001). World medical association declaration of Helsinki. Ethical principles for medical research involving human subjects. Bulletin of the World Health Organization, 79(4), 373-374.

Zakour, M. y Gillespie, D. (2012). Community disaster vulnerability: Theory, research, and practice. Springer. https://doi.org/10.1007/978-1-4614-5737-4

Zhou, C., Su, F., Pei, T., Zhang, A., Du, Y., Luo, B., ... Xiao, H. (2020). COVID-19: Challenges to GIS with big data. Geography and Sustainability, 1(1), 77-87. https://doi.org/10.1016/j.geosus.2020.03.005

\section{Breve CV de los autores}

\section{Tatiana Íñiguez-Berrozpe}

Profesora Ayudante Doctor en el área de Sociología del Departamento de Psicología y Sociología de la Universidad de Zaragoza (Facultad de Educación). Pertenece al Grupo de Investigación EDUCAVIVA (Grupo de Referencia financiado por el Gobierno de 
Aragón). Es editora adjunta de la Revista Internacional de Sociología de la Educación (RISE) y editora consultiva de Adult Education Quarterly (AEQ). Su principal área de investigación es la Sociología de la Educación, concretamente en los temas de: Educación de Personas Adultas, Aprendizaje Permanente y Convivencia Escolar, con los que ha participado y coordinado distintos proyectos regionales, nacionales y europeos, y publicado diversos artículos en revistas indexadas en Web of Science y Scopus. ORCID ID: https://orcid.org/0000-0003-4530-9645. E-mail: tatianai@unizar.es

\section{Raquel Lozano-Blasco}

Graduada en Magisterio de Educación Primaria e Educación Infantil, Máster en Estudios en Avanzados en el Lenguaje, la Comunicación y sus Patologías. Premio extraordinario de final grado, premio al mejor expediente de la Universidad de Zaragoza y premio nacional de final de grado. Matriculada en el programa de Doctorado de Educación de Zaragoza, goza de un contrato predoctoral FPU. ORCID ID: https://orcid.org/ooooo002-0100-1449. Email: rlozano@unizar.es

\section{Alberto Quílez-Robres}

Profesor Asociado en el área de Teoría e Historia de la Educación de la Universidad de Zaragoza y maestro de Educación Primaria y miembro del Equipo de Orientación en el colegio Sagrado Corazón Moncayo. Es Graduado en Maestro de Educación Primaria, Máster en Neuropsicología y Educación especializado en la rama de investigación por la Universidad Internacional de La Rioja y Doctorando por la Universidad de Zaragoza. Es colaborador del Grupo de Investigación EDUCAVIVA y del proyecto de innovación 12 Mirrors en la Universidad de Zaragoza. Sus investigaciones se centran en el estudio de las variables psicológicas vinculadas al rendimiento académico tratando de manera específica el desarrollo de la Funciones Ejecutivas y Factores de Inteligencia. ORCID ID: https://orcid.org/0000-0001-8473-8114. Email: aquilez@unizar.es

\section{Alejandra Cortés Pascual}

Profesora Titular en la Universidad de Zaragoza. España. Vicedecana de Innovación, Investigación y Comunicación de la Facultad de Educación de la Universidad de Zaragoza. Ha participado en diferentes proyectos (Gobierno de España) y ha coordinado varios proyectos regionales como investigadora principal. A nivel europeo actualmente participa en un proyecto Erasmus + (E-Tutoring), siendo la coordinadora en España (con casi 300,000 euros de financiación). Ha publicado en revistas de impacto nacionales e internacionales. Es coordinadora del máster en aprendizaje permanente: iniciación a la investigación. También directora de la Cátedra de Innovación Educativa de Juan de Lanuza de la Universidad de Zaragoza. Es investigadora principal de la Cátedra UNESCO de comunicación y valores. ORCID ID: https://orcid.org/0000-0002-2839-7041. Email: alcortes@unizar.es 